\title{
Quantitative Nano-characterization of Polymers Using Atomic Force Microscopy
}

\author{
Milad Radiom $\S^{\star a}$, Svilen Kozhuharova, Phally Kong ${ }^{\mathrm{b}}$, Michela di Giannantonio ${ }^{\mathrm{b}}$, Mathieu A. Ayer ${ }^{\mathrm{c}}$ \\ Plinio Maroni ${ }^{\mathrm{a}}$, Andreas F. M. Kilbinger ${ }^{\mathrm{b}}$, Katharina M. Fromm ${ }^{\mathrm{b}}$, Christoph Weder ${ }^{\mathrm{c}}$, and Michal \\ Borkovec ${ }^{a}$
}

§SCS-Metrohm Award for best oral presentation in Polymer, Colloids \& Interfaces

\begin{abstract}
The present article offers an overview on the use of atomic force microscopy (AFM) to characterize the nanomechanical properties of polymers. AFM imaging reveals the conformations of polymer molecules at solidliquid interfaces. In particular, for polyelectrolytes, the effect of ionic strength on the conformations of molecules can be studied. Examination of force versus extension profiles obtained using AFM-based single molecule force spectroscopy gives information on the entropic and enthalpic elasticities in $\mathrm{pN}$ to $\mathrm{nN}$ force range. In addition, single molecule force spectroscopy can be used to trigger chemical reactions and transitions at the molecular level when force-sensitive chemical units are embedded in a polymer backbone.
\end{abstract}

Keywords: AFM imaging · Atomic force microscopy · Mechanochemistry · Single molecule force spectroscopy · Single molecules

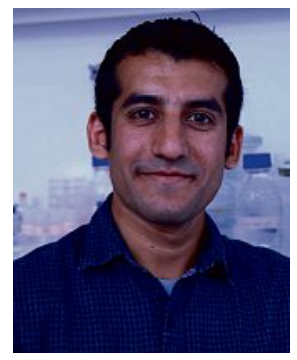

tory of Prof. Michal Borkovec to work on collaborative research activities within the Swiss National Center of Competence in Research Bio-Inspired Materials. His research includes single molecule measurements and molecular mechanochemistry.

Milad Radiom studied Mechanical Engineering at Amirkabir University of Technology (Iran) and Nanyang Technological University (Singapore) to respectively receive his $B S c$ and M.Eng degrees. He subsequently obtained his PhD in Chemical Engineering, under the supervision of Prof. William Ducker, at Virginia Tech (United States) in 2014. He then moved to University of Geneva (Switzerland) as a postdoctoral research associate in labora-

\footnotetext{
${ }^{*}$ Correspondence: Dr. M. Radiom ${ }^{\mathrm{a}}$

E-mail: milad.radiom@unige.ch

aDepartment of Inorganic and Analytical Chemistry University of Geneva

Sciences II

30, Quai Ernest-Ansermet

$\mathrm{CH}-1211$ Geneva 4

bDepartment of Chemistry

University of Fribourg

Chemin du Musée 9, $\mathrm{CH}$-1700 Fribourg

cAdolphe Merkle Institute

University of Fribourg

Chemin des Verdiers 4, CH-1700 Fribourg
}

\section{Introduction}

AFM was invented in 1986 as a highresolution imaging technique giving topographical information by means of tracking contact forces between an AFM tip and a sample surface. ${ }^{[1]}$ AFM enables us to visualize atoms and molecules and chemical bonds when imaged at low temperatures, ${ }^{[2]}$ as well as conformations of polymers in their various environments. ${ }^{[3,4]}$ For the latter studies, polymers are adsorbed from a dilute solution onto an atomically flat solid substrate such as mica, gold, or highly oriented pyrolytic graphite. Although early AFM images were obtained in contact mode, where a physical contact between AFM tip and sample was established, interest in imaging soft and destructible samples such as cells, DNA, proteins and various synthetic polyelectrolytes resulted in development of non-contact mode imaging techniques (Fig. 1). ${ }^{[5-8]}$ Analysis of AFM images provides useful information on conformational transitions, and enables one to extract various parameters, such as persistence length or gyration radius. ${ }^{[4,9,10]}$ These nanomechanical properties depend on chemical structure, line charge density of molecules, as well as solvent quality.[10-12]
A decade after the invention of the AFM, Gaub and coworkers showed that it can also be used to manipulate and to extend single polymer molecules. ${ }^{[13]}$ The research on muscle protein titin opened a new area of application for AFM, for which the term single molecule force spectroscopy (SMFS) was coined. SMFS experiments are realized by adsorbing a polymer film on solid substrate or by chemically binding a single polymer molecule to the tip of an AFM cantilever. The tip is then brought into contact with the substrate, which occasionally results in extending a single molecule (Fig. 1). Both the solid substrate and the AFM tip can be functionalized to tune various interactions at purpose, including desorption or extension. [14,15] To model such force-extension profiles the polymer chain is mimicked by a continuous curve or by a series of discrete segments that are freely jointed or that are jointed at a given angle, but allow for rotational and torsional degrees of freedom. ${ }^{[16]}$ These models normally incorporate a characteristic length corresponding to an entropic elasticity and a characteristic elasticity constant corresponding to the enthalpic contribution. SMFS was further combined with electrochemistry to obtain a nano-thermodynamic cycle by continuous extension-relaxation and oxidation-reduction processes ${ }^{[17]}$ and a correlation force spectrometer that allows to obtain time-resolved mechanical properties of single molecules. ${ }^{[18]}$

More recently, SMFS has entered the realm of mechanochemistry (Fig. 1). To obtain such information, special polymers 


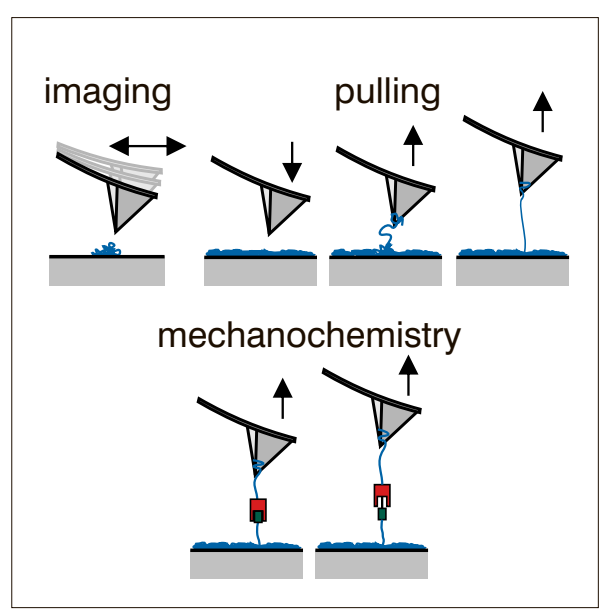

Fig. 1. Schematics of AFM non-contact mode imaging, single molecule pulling, and mechanochemistry.

are synthesized that contain along the backbone force-sensitive units, referred to as mechanophores. Application of mechanical force triggers transitions within the mechanophore units. One may also incorporate a functional group at one end of a polymer and investigate specific interactions between the functional group and surface. This approach leads to remarkable insights into forces and kinetics of various chemical reactions and transitions, including complexation and coordination bonds, ${ }^{[19,20]}$ receptor-donor interaction, ${ }^{[21]}$ hydrogen-bonding, ${ }^{[22]}$ and covalent bonds. ${ }^{23,24]}$ Mechanical force reduces the activation energy barrier, and thus facilitates the transitions by thermal activation. Without mechanical force, the transitions can only proceed by other triggers. An example is the isomerization of a carboncarbon double bond, which is ubiquitous in nature and technology, and is relevant, for example, in the isomerization of 11-cis-retinal to all-trans-retinal in the human eye, ${ }^{225]}$ or the isomerization of stilbene.[26] Catalysts such as silver, iodine and ruthenium have also been used to induce the isomerization of the double bond. ${ }^{[27]}$ As will be discussed below, isomerization of carbon-carbon double bonds can be activated mechanically at a force of about $800 \mathrm{pN}$.[28]

Let us now illustrate these notions with specific examples. The structures of polymers used in the experiments are summarized in Fig. 2. The polymers are poly(2-vinyl pyridine) (P2VP, molecular weight $M_{w}=150 \mathrm{Kg} / \mathrm{mol}$, and dispersity $Ð=1.1$ ), a poly(urethane) made from poly(tetrahydrofuran), 1,4-butanediol, and methylene diphenyl diisocyanate (PU, $\mathbf{M}_{\mathrm{w}}$ $=200 \mathrm{~kg} / \mathrm{mol}, Ð=1.6)$ and a triblock copolymer of poly(exo-N-(2-aminoethyl)5-norbornene-2,3-dicarboximide) and poly(exo-N-hexyl-5-norbornene-2,3-dicarboximide) $\left(\mathbf{P 1}, \mathbf{M}_{\mathrm{w}}=230 \mathrm{~kg} / \mathrm{mol}, \emptyset=\right.$ 1.6). P2VP was purchased from Sigma-
Aldrich. PU and P1 were synthesized by the methods reported in ref. [29] and ref. [30], respectively.

\section{Conformation of Polymer Molecules}

Conformation of single molecules is characterized by bond-bond correlation functions between tangent unit vectors $\mathbf{n}$ along the contour of the molecule. This correlation function normally decays on the order of $\mathrm{nm}$. The decay is exponential within the worm-like chain (WLC) model. For an adsorbed polymer, the characteristic decay length is related to the persistence length $l_{\mathrm{p}}$ of the molecule, where:

$$
\langle n(0) . n(s)\rangle=\exp \left(-\frac{s}{2 l_{p}}\right)
$$

For a charged molecule, the persistence length is normally attributed to an inherent backbone rigidity, which contributes to the bare persistence length, as well as an electrostatic term whose strength depends on the ionic level of the solution. Odijk, Skolnik and Fixman predicted a fast decay of the electrostatic contribution proportional to the inverse of the solution ionic level,[31,32] while a weaker decay was observed in experiments and simulations. ${ }^{[9,33,34]}$

Let us use AFM imaging to illustrate the conformational transition of a poly- electrolyte, namely P2VP, at two different ionic levels. At pH 3.0, P2VP is positively charged. Fig. 3 shows AFM images of P2VP in $1 \mathrm{mM}$ and $100 \mathrm{mM}$ solutions. To prepare the dilute films, $20 \mathrm{~mL}$ of $0.1 \mathrm{mg} / \mathrm{L}$ polymer solution was deposited on freshly cleaved mica for $40 \mathrm{~s}$. The polymer solution was then replaced with a larger volume of the electrolyte solution. The adsorbed molecules were imaged with a Cypher AFM (Asylum Research) in amplitude-modulation mode. Silicon tips (BL-AC40TS, Olympus) with nominal tip radius $<10 \mathrm{~nm}$, spring constant in the range of $0.07-0.15 \mathrm{~N} / \mathrm{m}$, and resonance frequency around $30 \mathrm{kHz}$ in water and $130 \mathrm{kHz}$ in air were used. Prior to imaging, the tip was treated in a UV-ozone cleaner (PSD Pro, Novascan, Ames, USA) for $20 \mathrm{~min}$ in an oxygen-enriched atmosphere. A scan rate of $4.88 \mathrm{~Hz}$ with free oscillation amplitude (FOA) of about $10 \mathrm{~nm}$ and amplitude setpoint of about $76 \%$ of FOA were also used. The imaging was carried out at a temperature of $25^{\circ} \mathrm{C}$.

The image at $1 \mathrm{mM}$ shows that the molecules assume extended random coil conformations, while at $100 \mathrm{mM}$, the molecules feature a collapsed coil conformation. The collapse of the molecule at higher ionic level is due to the screening of electrostatic repulsion between the charged monomers. This observation suggests that, at the lower ionic level, the electrostatic term greatly contributes to the conformational persistence in the molecules. Similar trends have been observed for P2VP as a function of solution $\mathrm{pH} .{ }^{[3]}$ It is possible to

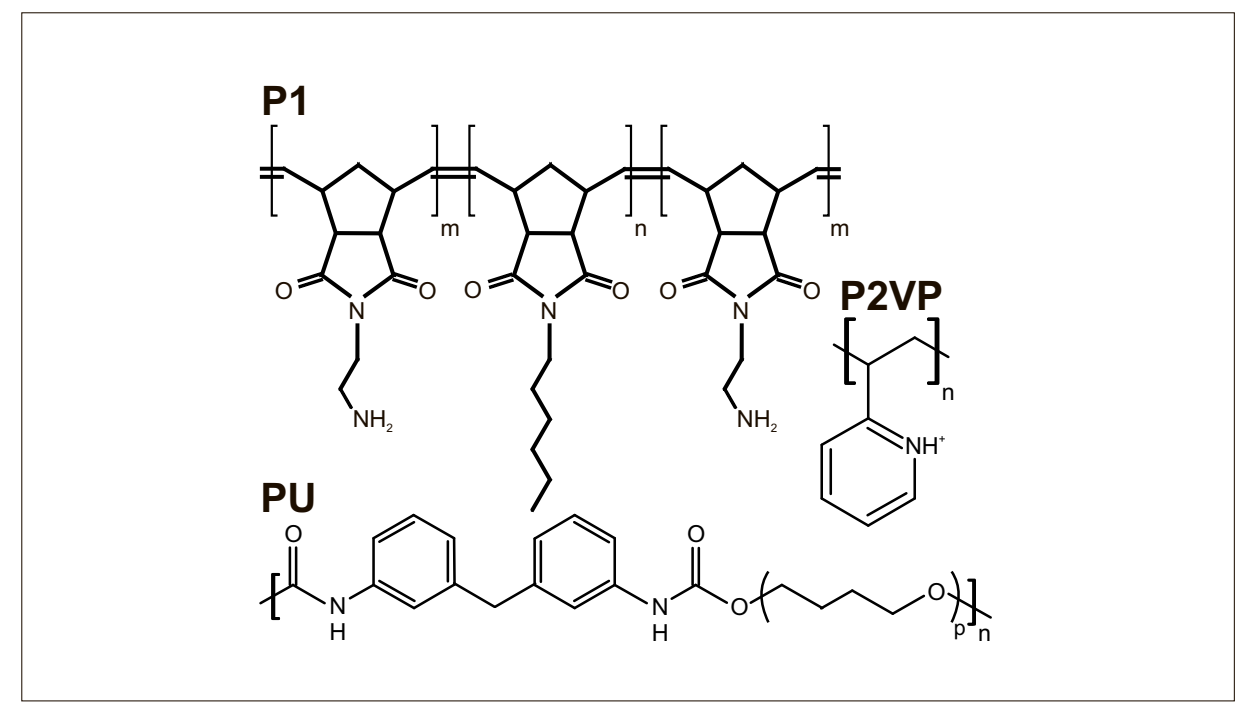

Fig. 2. The investigated polymers include poly(2-vinyl pyridine) (P2VP), a poly(urethane) made from poly(tetrahydrofuran), 1,4-butanediol, and methylene diphenyl diisocyanate (PU), ${ }^{[29]}$ and a triblock copolymer of poly(exo-N-(2-aminoethyl)-5-norbornene-2,3-dicarboximide) and poly(exo$\mathrm{N}$-hexyl-5-norbornene-2,3-dicarboximide) (P1) ${ }^{[30]}$ The side blocks of $\mathbf{P} \mathbf{1}$ are about 11 monomers long $(m \approx 11)$ while the middle block is about 544 monomers long $(n \approx 544)$. The side blocks contain amine. In the experiments using P1, the AFM tip was functionalized with epoxide. Amine enhances adhering of the polymer to AFM tip by covalently bonding with epoxide. The covalent bonding helps pull the polymer to elevated forces $(\sim 1 \mathrm{nN})$. 
quantify the persistence lengths of the adsorbed molecules using an image analysis software which computes the lateral coordinates of the molecules from which the correlation function is calculated. ${ }^{[35]}$ These results will be presented elsewhere.

\section{Molecular Elasticity}

Elasticity of polymers at the single molecule level is obtained from an analysis of their force versus extension profiles obtained from AFM pulling experiments. The loss of entropy as a consequence of increase in the end-to-end distance of the pulled molecule results in a restoring force that is sensed by the AFM probe. At forces in the $\mathrm{nN}$ range, chemical bonds and bond angles are also significantly distorted, which leads to an enthalpic contribution. In this case, the polymer molecule may extend beyond its contour length. ${ }^{[36]}$

The first step in the analysis is the identification of single molecule events that are originating from molecules that are firmly anchored on both ends to the solid substrate and the AFM tip. While a variety of
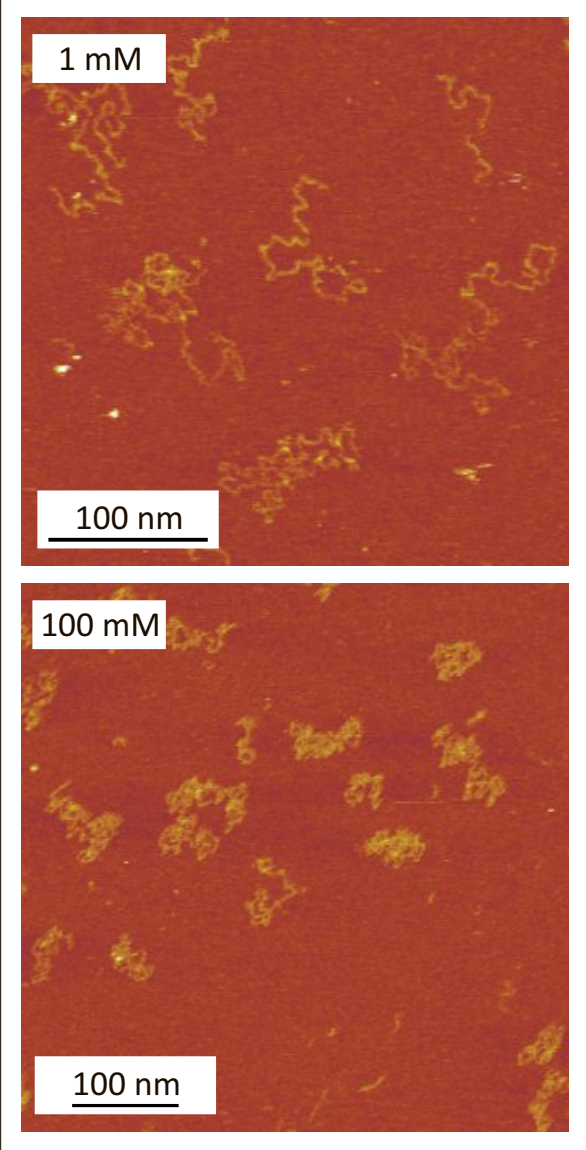

Fig. 3. AFM images of P2VP adsorbed on mica at different ionic levels $1 \mathrm{mM}$ and 100 $\mathrm{mM}$, both at $\mathrm{pH}$ 3.0. At these conditions P2VP is positively charged. At low ionic level the molecules form an extended random coil while at high ionic level they collapse. approaches exist, the most reliable ones are the nano-handling of thick molecules, ${ }^{37]}$ and a statistical approach that consists of normalization of the extension length of the pulled polymers and overlapping of the force versus normalized extension profiles. When the force versus normalized extension profiles overlap, single molecule extension responses are likely. In the latter case, if more than one statistical population exists, one can usually confirm the population that corresponds to stretching of single molecules with firmly anchored ends. This is done by repeating the experiments on a more dilute polymer layer, or on surfaces that are chemically different. ${ }^{[28]}$

The freely jointed chain (FJC) model can describe the force versus extension profiles of single molecules:[38]

$$
x=L\left[\operatorname{coth}\left(\frac{l_{K} F}{k T}\right)-\frac{k T}{l_{K} F}+\frac{F}{K}\right] \text { (2) }
$$

where $L$ is the contour length, $k$ the Boltzmann constant, $T$ the absolute temperature. The Kuhn length $l_{\mathrm{K}}$, and the force constant $K$ represent the nanomechanical properties of single molecules in the FJC model. This model has been successfully used in describing the force versus extension profiles of synthetic polymers. ${ }^{[38]}$

Fig. 4 shows the force versus extension profiles of P2VP and PU. Experiments with P2VP were performed in $1 \mathrm{mM}$, pH 3.0 solution and on mica while experiments with PU were carried out in dimethylsulfoxide (DMSO) and on silica. In both cases, polymer solutions of $100 \mathrm{mg} / \mathrm{L}$ concentration were used. The deposition period for P2VP was $40 \mathrm{sec}$ and for PU, $2 \mathrm{~h}$. For P2VP the polymer solution was exchanged with a larger volume of the electrolyte solution while for $\mathbf{P U}$, the polymer film was initially rinsed with DMSO and then dried with a stream of nitrogen prior to the force spectroscopy experiments. The normalization procedure was used to obtain single molecule extensions. Thereafter, FJC model was used to fit the individual force versus extension profiles. An average Kuhn length and force constant was obtained for each polymer. These values were $l_{\mathrm{K}}$ $=0.52 \pm 0.07 \mathrm{~nm}$ and $K=9.5 \pm 0.2 \mathrm{nN}$ for P2VP and $0.30 \pm 0.02 \mathrm{~nm}$ and $27.1 \pm 3.3 \mathrm{nN}$ for PU. These results suggest that at low forces, $\mathbf{P U}$ is more flexible than $\mathbf{P 2 V P}$ due to its lower Kuhn length. At high forces, PU becomes stiffer than P2VP as a result of its higher force constant.

\section{Molecular Mechanochemistry}

Cis to trans isomerization of a carbon-carbon double bond has an activation energy $E_{\mathrm{b}}=170 \mathrm{~kJ} \mathrm{~mol}^{-1}$ and a thermal rate of generally less than $10^{-8} \mathrm{~s}^{-1}$. [39] The high energy barrier and low rates hinder the isomerization to proceed thermally at room temperature. Using AFM, we pulled molecules which contained cis double bond units in the backbone and obtained their force versus extension profiles. The force is transferred to the units via the polymer backbone, and results in reduction of the energy barrier by an amount $F \Delta x$, where $F$ is the force and $\Delta x$ the displacement of cis monomers along the reaction coordinate. Occasionally, the energy barrier is lowered enough and isomerization occurs through thermal activation. In the force versus extension profiles of $\mathbf{P 1}$ (Fig. 5(a)), isomerization is identified by a sudden increase in the extension of the molecule. The extension increases since trans isomers are longer than cis isomers. The force where the isomerization occurs is denoted by the isomerization force $F_{\text {ct }}$. The force versus extension response of $\mathbf{P 1}$ differs from those of force-insensitive polymers such as P2VP and PU introduced in the previous section. In the latter, the force increases with extension until the molecule breaks from the AFM tip or the solid substrate. In the former, the molecule is tethered between the AFM tip and the solid substrate even after the isomerization.

Here again application of the normalization procedure is important to identify single molecule events, see Fig. 5(b). The force versus extension profiles are fitted to the FJC model (Eqn. (2)) which results in the nanomechanical properties, Kuhn length $l_{\mathrm{K}}$ and force constant $K=20 \pm 3 \mathrm{nN}$, of P1. Analysis of the single molecule isomerization events results in an isomerization force $F_{\mathrm{ct}}=800 \pm 60 \mathrm{nN}$. [28] The isomerization force is lower than the force of breaking of covalent bonds and rings, $1-2 \mathrm{nN}$, which have higher activation energies. ${ }^{[40,41]}$

\section{Conclusion}

AFM has revolutionized our analytical capabilities of polymers and enabled high resolution and nanoscale description of individual molecules. By adsorbing polyelectrolytes from dilute solutions on appropriate solid substrates and imaging their contour, the dependence of their conformation on the ionic level of solution can be obtained. A similar preparation can be used to pick and then pull single molecules, which on the other hand reveals their molecular elasticity. By incorporation of force-sensitive units in polymer backbones, AFM can be used to activate and probe chemical transitions within individual molecules. 


\section{Acknowledgement}

The research leading to these results has received funding from the National Center of Competence in Research (NCCR) for BioInspired Materials, University of Geneva, and the European Research Council under the European Union's Seventh Framework Programme (FP7/2007-2013)/ERC grant agreement $\mathrm{n}^{\circ}$ 291490-MERESPO.

Received: December 1, 2016
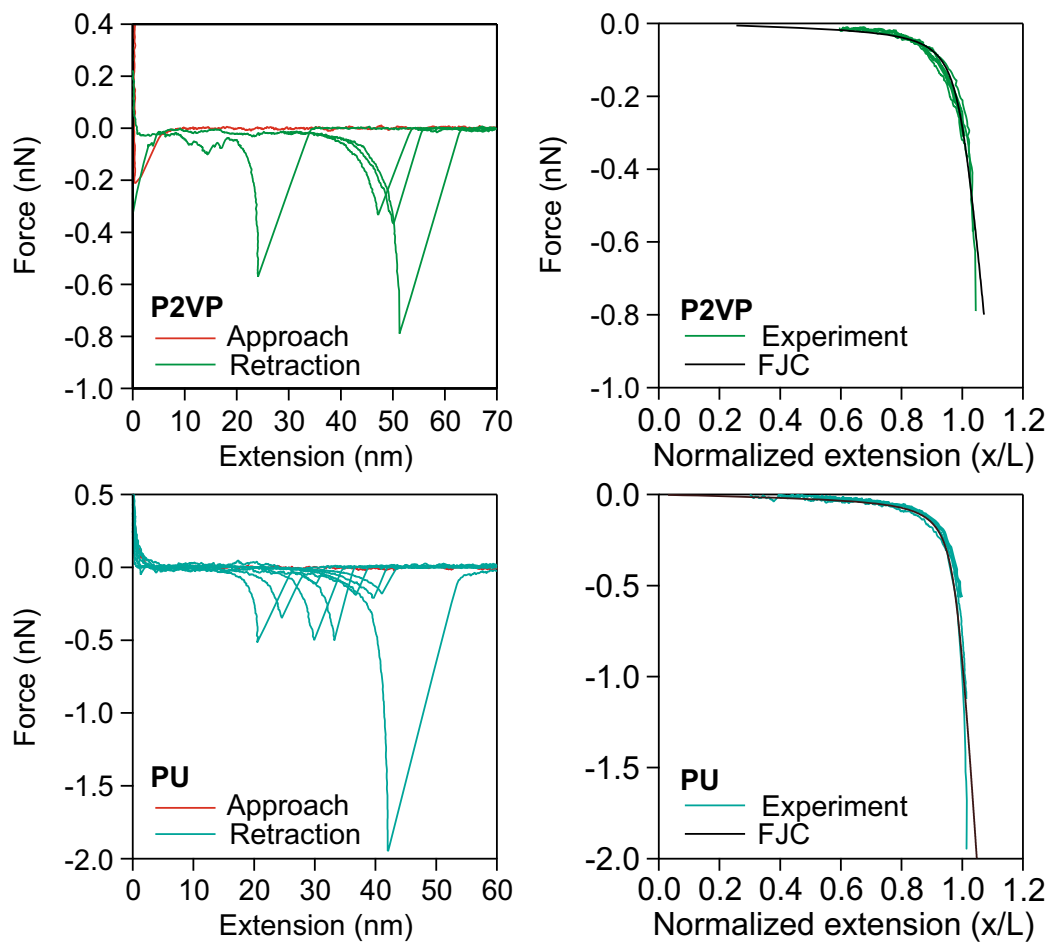

Fig. 4. Molecular elasticity of P2VP and PU obtained from single molecule pulling. Experiments with P2VP were performed in $1 \mathrm{mM}$, pH 3.0 solution. Experiments with PU were performed in dimethylsulfoxide (DMSO). Using the FJC model, a persistence length $I_{\mathrm{K}}=0.52 \pm 0.07 \mathrm{~nm}$ and a force constant $K=9.5 \pm 0.2 \mathrm{nN}$ were obtained for P2VP. These values were respectively, $0.30 \pm 0.02$ $\mathrm{nm}$ and $27.1 \pm 3.3 \mathrm{nN}$ for $\mathbf{P U}$. (a)

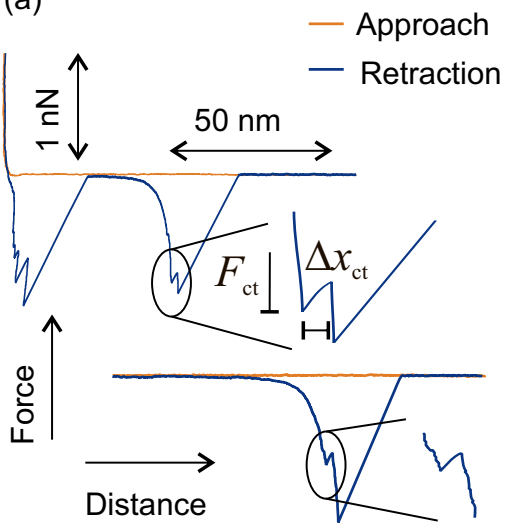

(b)

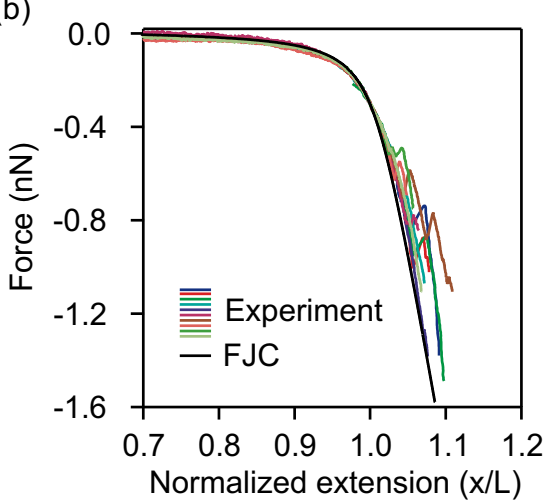

Fig. 5. (a) Force versus extension profiles of $\mathbf{P} 1$ showing isomerization events. The onset of cis-totrans isomerization is denoted by the isomerization force that accompanies an extension increase. (b) Force versus normalized extension profiles of $\mathbf{P 1}$ together with the post-isomerization sections. Using the FJC model, a persistence length $I_{\mathrm{K}}=0.53 \pm 006 \mathrm{~nm}$ and a force constant $\mathrm{K}=20 \pm 3 \mathrm{nN}$.
[7] J. V. Lauritsen, M. Reichling, J. Phys. Condes. Matter 2010, 22, 263001.

[8] D. J. Marchand, E. Hsiao, S. H. Kim, Langmuir 2013, 29, 6762

[9] L. Grebikova, S. Kozhuharov, P. Maroni, A. Mikhaylov, G. Dietler, A. D. Schluter, M Ullner, M. Borkovec, Nanoscale 2016, 8, 13498.

[10] Y. Roiter, O. Trotsenko, V. Tokarev, S. Minko, J. Am. Chem. Soc. 2010, 132, 13660.

[11] A. Kiriy, G. Gorodyska, S. Minko, W. Jaeger, P. Stepanek, M. Stamm, J. Am. Chem. Soc. 2002, $124,13454$.

[12] Y. Roiter, W. Jaeger, S. Minko, Polymer 2006 47, 2493.

[13] M. Rief, M. Gautel, F. Oesterhelt, J. M. Fernandez, H. E. Gaub, Science 1997, 276, 1109.

[14] L. Grebikova, M. Radiom, P. Maroni, D. A. Schlüter, M. Borkovec, Polymer 2016, 102, 350.

[15] M. Geisler, R. R. Netz, T. Hugel, Angew. Chem. Int. Ed. 2010, 49, 4730.

[16] L. Livadaru, R. R. Netz, H. J. Kreuzer, Macromolecules 2003, 36, 3732.

[17] W. Q. Shi, M. I. Giannotti, X. Zhang, M. A. Hempenius, H. Sconherr, G. J. Vancso, Angew. Chem., Int. Ed. 2007, 46, 8400.

[18] M. Radiom, M. R. Paul, W. A. Ducker, Nanotechnology 2016, 27, 255701.

[19] T. Auletta, M. R. de Jong, A. Mulder, F. van Veggel, J. Huskens, D. N. Reinhoudt, S. Zou, S. Zapotoczny, H. Schonherr, G. J. Vancso, L. Kuipers, J. Am. Chem. Soc. 2004, 126, 1577.

[20] S. Kado, K. Kimura, J. Am. Chem. Soc. 2003, 125,4560 .

[21] H. Skulason, C. D. Frisbie, J. Am. Chem. Soc. $2002,124,15125$.

[22] A. Embrechts, A. H. Velders, H. Schoenherr, G. J. Vancso, Langmuir 2011, 27, 14272.

[23] D. Schuetze, K. Holz, J. Mueller, M. K. Beyer, U. Luening, B. Hartke, Angew. Chem. Int. Ed. 2015, 54, 2556

[24] H. M. Klukovich, T. B. Kouznetsova, Z. S. Kean, J. M. Lenhardt, S. L. Craig, Nature Chem. 2013, 5, 110.

[25] K. Palczewski, J. Biol. Chem. 2012, 287, 1612.

[26] R. E. Dawson, S. F. Lincoln, C. J. Easton, Chem. Commun. 2008, 3980.

[27] S. K. Sharma, V. K. Srivastava, R. V. Jasra, J. Mol. Catal. A-Chem. 2006, 245, 200.

[28] M. Radiom, P. Kong, P. Maroni, M. Schafer, A Kilbinger, M. Borkovec, Phys. Chem. Chem. Phys. 2016, 18, 31202.

[29] M. A. Ayer, Y. C. Simon, C. Weder, Macromolecules 2016, 49, 2917

[30] M. Schaefer, N. Hanik, A. F. M. Kilbinger, Macromolecules 2012, 45, 6807.

[31] T. Odijk, J. Polym. Sci. B 1977, 15, 477.

[32] J. Skolnick, M. Fixman, Macromolecules 1977, 10, 944

[33] R. R. Netz, H. Orland, Eur. Phys. J. B 1999, 8,81 .

[34] M. Ullner, J. Phys. Chem. B 2003, 107, 8097.

[35] A. Mikhaylov, S. Sekatskii, G. Dietler, J. Adv Microscopy Res. 2013, 8, 241.

[36] T. Hugel, M. Rief, M. Seitz, H. E. Gaub, R. R. Netz, Phys. Rev. Lett. 2005, 94.

[37] L. Grebikova, P. Maroni, B. Z. Zhang, D. A. Schlüter, M. Borkovec, ACS Nano 2014, 8 , 2237.

[38] M. I. Giannotti, G. J. Vancso, ChemPhysChem 2007, 8, 2290 .

[39] J. L. Magee, W. Shand, H. Eyring, J. Am. Chem. Soc. 1941, 63, 677.

[40] J. Wang, T. B. Kouznetsova, Z. Niu, M. T. Ong, H. Klukovich, A. L. Rheingold, T. J. Martinez, S. L. Craig, Nature Chem. 2015, 7, 323

[41] M. Grandbois, M. Beyer, M. Rief, H. ClausenSchaumann, H. E. Gaub, Science 1999, 283, 1727. 